\title{
ON THE EXISTENCE OF THE RESOLVENT KERNEL FOR ELLIPTIC DIFFERENTIAL OPERATOR IN A COMPACT RIEMANN SPACE
}

\author{
KÔSAKU YOSIDA
}

\$1. Introduction. We consider the differential operator

$$
(A f)(x)=b^{i j}(x) \frac{\partial^{2} f}{\partial x^{i} \partial x^{j}}+a^{i}(x) \frac{\partial f}{\partial x^{i}}+c(x) f(x)
$$

in an $n$-dimensional $(n \gtrsim 2)$, orientiable, compact Riemann space $R$ with the metric $d s^{2}=g_{i j}(x) d x^{i} d x^{j}$. Here $b^{i j}(x)$ is a contravariant tensor such that the quadratic form $b^{i j}(x) \xi_{i} \xi_{j}$ is $>0$ for $\sum_{i=1}^{n} \xi_{i}^{2}>0$, and $a^{i}(x)$ changes, by the coordinates transformation $x \rightarrow \bar{x}$, as follows:

$$
\bar{a}^{i}(\bar{x})=\frac{\partial \bar{x}^{i}}{\partial x^{k}} a^{k}(x)+\frac{\partial^{2} \bar{x}^{i}}{\partial x^{j} \partial x^{s}} b^{j s}(x) .
$$

These transformation rules for the coefficients are connected with the fact that the value of $(A f)(x)$ is independent of the local coordinates $\left(x^{1}, \ldots, x^{n}\right)$.

For the sake of simplicity, we assume that $R$ is an infinitely differentiable manifold and that $g_{i j}(x), b^{i j}(x), a^{i}(x), c(x)$ are infinitely differentiable functions of the local coordinates $\left(x^{1}, \ldots, x^{n}\right)$. We consider $A$ as an additive operator whose domain $D(A)$ is the totality of real-valued infinitely differentiable functions on $R$, with values in the Banach space $C(R)$ of the totality of real-valued continuous functions $f(x)$ on $R$, metrized by the norm $\|f\|=\max _{x \in R}|f(x)|$. As in a preceding note, ${ }^{1)}$ we may prove $(\S 2)$ the folowing existence theorem:

Let us consider $D(A)$ as a linear subspace of $C(R)$ and let $\tilde{A}$ be the smallest closed extension of the operator $A$. Then, if

$$
m>\max _{x}|c(x)|,
$$

the operator $\left(I-m^{-1} \tilde{A}\right)(I=$ the identity operator) admits a bounded linear inverse, the resolvent $I_{m}=\left(I-m^{-1} \tilde{A}\right)^{-1}$ defined on $C(R)$.

Received September 25, 1951.

1) K. Yosida: Integrability of the backward diffusion equation in a compact Riemannian space, Nagoya Math. Journal, Vol.3, 1-4 (1951). At this juncture, the author wishes to correct the errata in the cited paper. $\left(-m^{-1} \widetilde{A}\right)$ on page 3 , line 2 must be corrected as $\left(I-m^{-1} \widetilde{A}\right), \quad D(A)$ and $A$ on page 3, line 5 must be corrected as $D\left(I_{m}\right)$ and $I_{m b}$ respecteively. 
The purpose of the present note is to show that this resolvent may, for sufficiently large $m$, be represented as an integral operator of the form

$$
\begin{aligned}
& \left(I_{m} f\right)(x)=\int_{R} p_{m}(x, y) f(y) d y, \quad d y=\sqrt{g(x)} d x^{1} \ldots d x^{n}, \\
& g(x)=\operatorname{det}\left(g_{i j}(x)\right),
\end{aligned}
$$

with a measurable kernel $p_{m}(x, y)$. The result will be applied to the explicite expression for the transition probability of the stochastic process defined by the diffusion equation

$$
\frac{\partial f}{\partial t}=A f \quad(t \geqslant 0) .
$$

§2. The existence of the resolvent $\boldsymbol{I}_{m}$. We will prepare lammas.

Lemma 1. Let $m$ satisfy (1.3) and let $\left(\left(I-m^{-1} A\right) f\right)(x)=g(x)$ for $f \in D(A)$. Then we have

$$
\begin{aligned}
\max _{x} g(x) & \geqq\left(1-m^{-1}\|c\|\right) \max _{x} f(x) \text { for } \max _{x} f(x) \geqq 0 \\
& \geqq\left(1-m^{-1}\left(\min _{x} c(x)\right) \max _{x} f(x) \text { for } \max _{x} f(x) \leqq 0,\right. \\
\min _{x} g(x) & \leqq\left(1-m^{-1}\|c\|^{\prime} \min _{x} f(x) \text { for } \min _{x} f(x) \leqq 0\right. \\
& \leqq\left(1-m^{-1}\left(\min _{x} c(x)\right) \min _{x} f(x) \text { for } \min _{x} f(x) \geqq 0 .\right.
\end{aligned}
$$

Proof. Let $f(x)$ reach its maximum and minimum at $x=x_{1}$ and $x_{2}$. Then we have, by

$$
b^{i j}(x) \frac{\partial^{2} f}{\partial x^{i} \partial x^{j}} \leqq 0 \quad\left(\text { at } x=x_{1}\right), \quad b^{i j}(x) \frac{\partial^{2} f}{\partial x^{i} \partial x^{j}} \geqslant 0 \quad\left(\text { at } x=x_{2}\right),
$$

the inequalities

$$
f\left(x_{1}\right)-m^{-1} c\left(x_{1}\right) f\left(x_{1}\right) \leqq g\left(x_{1}\right), \quad f\left(x_{2}\right)-m^{-1} c\left(x_{2}\right) f\left(x_{2}\right) \geqq g\left(x_{2}\right) .
$$

Lemma 2. The smallest closed extension $\tilde{A}$ of $A$ exists. It is defined as follows: $\tilde{A} f=e$ if there exists $\left\{f_{k}\right\} \subseteq D(A)$ such that the strong $\lim _{k \rightarrow \infty} f_{k}=f$, strong $\lim _{k \rightarrow \infty} A f_{k}=e$. Here strong lim means the lim defined by the norm of $C(R)$.

Proof. By the integral theorem of Green, we have

$$
\begin{aligned}
\int_{R}\left(A f_{k}\right)(x) h(x) d x & =\int_{R} f_{k}(x)\left(A^{\prime} h\right)(x) d x, \quad h \in D(A), \quad \text { where } \\
\left(A^{\prime} h\right)(x) & =\frac{1}{\sqrt{g(x)}} \frac{\partial^{2}}{\partial x^{i} \partial x^{j}}\left(\sqrt{g(x)} b^{i j}(x) h(x)\right) \\
& -\frac{1}{\sqrt{g(x)} \partial x^{i}}\left(\sqrt{g(x)} a^{i}(x) h(x)\right) \\
& +c(x) h(x)=\left(A_{1} h\right)(x)+c(x) h(x) .
\end{aligned}
$$


Thus, if strong $\lim _{k \rightarrow \infty} f_{k}=0$, we would have

$$
\int_{R} e(x) h(x) d x=\lim _{k \rightarrow \infty} \int_{R}\left(A f_{k}\right)(x) h(x) d x=\lim _{k \rightarrow \infty} \int_{R} f_{k}(x)\left(A^{\prime} h\right)(x) d x=0 .
$$

Hence we must have $e(x) \equiv 0$ for strong $\lim _{k \rightarrow \infty} f_{k}=0$. Therefore $\tilde{A} f$ is a onevalued function of $f$. independent of the sequence $\left\{f_{k}\right\}$ which defines $f$.

Lemma 3. The range $\left\{\left(I-m^{-1} A\right) f ; f \in D(A)\right\}$ is strongly dense in $C(R)$.

Proof. If otherwise, there would exists a measure $\mu(E)$, countably additive for Borel set $E$ of $R$, such that

the total variation of $\mu$ on $R$ is $\neq 0$,

$$
\int_{R}\left(\left(I-m^{-1} A\right) f\right)(x) \mu(d x)=0 \text { for } f \in D(A) .
$$

Since the operator $\left(I-m^{-1} A\right)$ is elliptic, there must exist ${ }^{21}$ infinitely differentiable function $h(x)$ such that

$$
\mu(E)=\int_{E} h(x) d x, \quad\left(\left(I-m^{-1} A^{\prime}\right) h\right)(x)=0 .
$$

Let $^{3)} k(x)$ be $=1,=-1$ or $=0$ according as $h(x)>0,<0$ or $=0$.

Then we have

$$
\begin{aligned}
0=\int_{R} k(x)\left(\left(I-m^{-1} A^{\prime}\right) h\right)(x) d x \geqslant \int_{R}\left(1-m^{-1}\|c\|\right)|h(x)| d x \\
-m^{-1} \sum_{i} \int_{P_{i}}\left(A_{1} h\right)(x) d x+m^{-1} \sum_{j} \int_{N_{j}}\left(A_{1} h\right)(x) d x,
\end{aligned}
$$

where $P(N)$ are connected domains in which $h(x)>0(<0)$ such that $h(x)$ vanishes on the boundaries $\partial P(\partial N)$. We have, by Green's integral theorem,

$$
\int_{P_{i}}\left(A_{1} h\right)(x) d x=\int_{\partial P_{i}} \frac{\partial h}{\partial} d S,
$$

where $n$ and $d S$ denote outer normal and positive measure on $\partial P$ respectively. Hence $\int_{P_{i}}\left(A_{1} h\right)(x) \leqq 0$. Similarly we have $\int_{x_{j}}\left(A_{i} h\right)(x) d x \geqq 0$. Thus we must have $h(x) \equiv 0$ and hence $\mu(E)=\int_{E} h(x) d x=0$, contrary to (2.4). Q.E.D.

We have incidentally proved the following lemma, which plays an important role in $\S 4$ below.

LEMma 4. For any $h \in D(A)$, we have, for sufficiently large $m$,

L. Schwartz: Théorie des distributions, Paris (1950).

3) Cf. K. Yosida: Integration of Fokker-Planck's equation with a boundary condition, Journal of the Math. Soc. of Japan, Vol.3, No. 1, 69-73 (1951). 


$$
\int_{R}\left|\left(\left(I-m^{-1} A\right) h\right)(x)\right| d x \gtrsim \frac{1}{2} \int_{R}|h(x)| d x .
$$

By the above three lemmas 1,2 and 3 , we see that, for $m>\|c\|$, the resolvent

$$
I_{m}=\left(I-m^{-1} \tilde{A}\right)^{-1}
$$

exists as a bounded linear operator on $C(R)$. Moreover, by lemma 1, the operator $I_{m}$ is positive :

$$
g(x) \geq 0 \text { on } R \text { implies } f(x)=\left(\left(I-m^{-1} \tilde{A}\right)^{-1} g\right)(x) \geq 0 \text { on } R .
$$

Hence, for fixed $x_{0} \in R,\left(I_{m} g\right)\left(x_{0}\right)$ is a bounded linear functional on $C(R)$ and thus

$$
\left(I_{m} g\right)\left(x_{0}\right)=\int_{R} P_{m}\left(x_{0}, d y\right) g(y),
$$

where $P_{m}\left(x_{0}, E\right)$ is a non-negative set function, countably additive for Borel set E. $P_{m}(x, E)$ is also Borel measurable in $x$ for fixed $E$.

We will show $(\S 4)$ that, for sufficiently large $m$,

$$
\begin{gathered}
P_{m}(x, E)=\int_{E} P_{m}(x, y) d y \text {, with a measurable density } P_{m}(x, y) \\
\text { satisfying certain regularity conditions (see (4.12) below) }
\end{gathered}
$$

To this purpose, we need a parametrix in the large, viz. almost Green's function of the operator $\left(I-m^{-1} A^{\prime}\right)$. This will be introduced in the next $\S$.

§3. The parametrix in the large. We adopt a new metric

$$
d r^{2}=b_{i j}(x) d x^{i} d x^{j},
$$

where $\left(b_{i j}(x)\right)$ is the inverse matrix of the matrix $\left(b^{i j}(x)\right)$. We also assume that the local coordinates $\left(x^{1}, \ldots, x^{n}\right)$ are a normal coordinates in the vicinity of the point $P=(0, \ldots, 0)$. Thus the adjoint poerator $A^{\prime}$ of $A$ is of the form $\left(b(x)=\operatorname{det}\left(b_{i j}(x)\right)\right)$ :

$$
\begin{aligned}
\left(A^{\prime} f\right)(x) & =\frac{1}{\sqrt{b(x)}} \frac{\partial^{2}}{\partial x^{i} \partial x^{j}}\left(\sqrt{b(x)} b^{i j}(x) f(x)\right) \\
& -\frac{1}{\sqrt{b(x)}} \frac{\partial}{\partial x^{2}}\left(\sqrt{b(x)} a^{i}(x) f(x)\right) \\
& +c(x) f(x) \\
& =(\Delta f)(x)+e^{i}(x) \frac{\partial f}{\partial x^{i}}+k(x) f(x), \text { where } \\
(\Delta f)(x) & =b^{i j}(x)\left[\frac{\partial^{2} f}{\partial x^{i} \partial x^{j}}-\frac{\partial f}{\partial x^{\alpha}}\left\{\begin{array}{l}
\alpha \\
i j
\end{array}\right\}\right] \text { (the Laplacian), } \\
\left\{\begin{array}{l}
\alpha \\
i j
\end{array}\right\} & =\frac{1}{2} b^{\alpha \mu}\left[\frac{\partial b_{\mu i}}{\partial x^{j}}+\frac{\partial b_{j \mu}}{\partial x^{i}}-\frac{\partial b_{i j}}{\partial x^{u}}\right] .
\end{aligned}
$$

Let $I=r^{2}$ be the square of the geodesic distance of the point $Q=\left(x^{1}, \ldots, x^{n}\right)$ 
from the point $P=(0, \ldots, 0)$. We have the well-known identity

$$
\begin{aligned}
& \Gamma=\Gamma_{P Q}=r^{2}=r_{P Q}^{2}=b_{\alpha \beta}(0) x^{\alpha} x^{\beta}, \\
& b_{\alpha \sigma}(x) x^{\beta}=b_{\alpha o}(0) x^{\sigma}, \\
& \left\{\begin{array}{l}
k \\
i j
\end{array}\right\} x^{i} x^{j}=0 .
\end{aligned}
$$

Let $\emptyset(\Gamma)$ be a function of $\Gamma=\Gamma_{P Q}^{2}$. Then, from

$$
\frac{\partial \Phi}{\partial x^{\alpha}}=\frac{d \Phi}{d \Gamma} \frac{\partial \Gamma}{\partial x^{\alpha}}, \frac{\partial^{2} \Phi}{\partial x^{\alpha} \partial x^{3}}=\frac{d^{2} \Phi}{d \Gamma^{2}} \frac{\partial \Gamma}{\partial x^{\alpha}} \frac{\partial \Gamma}{\partial x^{\beta}}+\frac{d \Phi}{d \Gamma} \frac{\partial^{2} \Gamma}{\partial x^{\alpha} \partial x^{3}},
$$

we obtain

$$
\left(A^{\prime} \Phi\right)(x)=\frac{d^{2} \Phi}{d \Gamma^{2}} b^{\alpha \beta}(x) \frac{\partial \Gamma}{\partial x^{\alpha}} \frac{\partial \Gamma}{\partial x^{3}}+\frac{d \Phi}{d \Gamma} \Delta \Gamma+\frac{d \Phi}{d \Gamma} e^{\alpha}(x) \frac{\partial \Gamma}{\partial x^{\alpha}}+k(x) \Phi(\Gamma) .
$$

The coefficients in this equation may be simplified as follows.) ${ }^{4}$ From (3.3)

$$
b^{\alpha \beta} \frac{\partial \Gamma \partial \Gamma}{\partial x^{\alpha} \partial x^{\beta}}=4 b^{\alpha \beta} b_{\alpha \sigma}(0) x^{\beta} b_{\beta \tau}(0) x^{\tau}=4 b^{\alpha \beta} b_{\alpha \sigma} x^{\sigma} b_{\beta \tau}(0) x^{\tau}=4 \Gamma \text {. }
$$

From (3.3) and the definition of the Laplacian in (3.2),

$$
\Delta \Gamma=2 b^{\alpha \beta} b_{\alpha \beta}(0)-2 b^{\alpha \beta} x^{\sigma} \frac{\partial b_{\alpha \pi}}{\partial x^{\beta}}+b^{\alpha \beta} x^{\sigma} \frac{\partial b_{\alpha \beta}}{\partial x^{\beta}}=2 n+x^{\sigma} \frac{\partial \log b}{\partial x^{\sigma}} \text {. }
$$

The last equality may be obtained by differentiating the 2 nd identity of (3.3) with respect $x^{\beta}$ and summing on the indices $\alpha$ and $\beta$ :

$$
b^{\alpha \beta} x^{\sigma} \frac{\partial b_{\alpha \sigma}}{\partial x^{\beta}}=-n+b^{\alpha \beta} b_{\alpha \beta}(0) \text {. }
$$

Therefore we have

$$
\left(A^{\prime} \Phi\right)(x)=4 \Gamma \frac{d^{2} \Phi}{d \Gamma^{2}}+\left[2 n+x^{3} \frac{\partial \log b}{\partial x^{\sigma}}+2 e^{\alpha} b_{\alpha \beta}(0) x^{\beta}\right] \frac{d \Phi}{d \Gamma}+k \Phi
$$

Thus, by taking

$$
\begin{aligned}
\Phi_{m}\left(\Gamma_{P Q}\right) & =-\frac{m}{2 \pi} \log r_{P Q}, \quad(n=2), \\
& =\frac{m}{N} r_{P Q}^{2-n}, \quad N=(n-2) 2(\pi)^{n / 2} / \Gamma(n / 2), \quad(n \geqq 3),
\end{aligned}
$$

we have

$$
\begin{aligned}
\left(A^{\prime} \Phi_{m}\right)(x) & =-\frac{m}{2 \pi}\left\{\frac{1}{2}\left(x^{\sigma} \frac{\partial \log b}{\partial x^{\sigma}}+2 e^{\alpha} b_{\alpha \beta}(0) x^{\beta}\right) r^{-2}+k \log r\right\}, \quad(n=2), \\
& =\frac{m}{N}\left\{\left(\frac{2-n}{2}\right)\left(x^{\sigma} \frac{\partial \log b}{\partial x^{\sigma}}+2 e^{\alpha} b_{\alpha \beta}(0) x^{\beta}\right) r^{-n}+k r^{2-n}\right\}, \quad(n \geqq 3) .
\end{aligned}
$$

4) We follow T. Y. Thomas and E. W. Titt: On the elementary solution of the general linear differential equation of the second order with analytic coefficients, Journal de Math., tome 18, 217-248 (1939). 
Hence (3.6) is a parametrix in the large of the operator $\left(I-m^{-1} A^{\prime}\right)$ in the following sense. By the integral theorem of Green $\left(d x=\sqrt{b(x)} d x^{1} \ldots d x^{n}\right)$, we obtain

$$
\begin{aligned}
\int_{D} h(x) & \left(\left(I-m^{-1} A\right) f\right)(x) d x-\int_{D} f(x)\left(\left(I-m^{-1} A^{\prime}\right) h(x) d x\right. \\
& =m^{-1} \int_{D}\left(f(x)\left(A^{\prime} h\right)(x)-h(x)(A f)(x)\right) d x \\
& =-m^{-1} \int_{\partial D}\left\{f \frac{\partial h}{\partial \nu}-h \frac{\partial f}{\partial \nu}+L f h\right\} d S,
\end{aligned}
$$

where $\nu$ is the inner transversal direction defined by

$$
\frac{d x^{j}}{\sqrt{b(x)} b^{i j}(x) \cos \left(n, x^{i}\right)}=d \nu(n \text { denotes the inner normal }),
$$

and $d S$ is the hypersurface element on the boundary $\partial D$ which surrounds the point $P=(0, \ldots, 0)$, and $L$ is a function continuous for $P=(0, \ldots, 0)$. If we take $\Phi_{m}\left(\Gamma_{P Q}\right)$ for $h(x)$ and the geodesic sphere of radius $\delta$ and $P=(0, \ldots, 0)$ as centre for $\partial D$, we obtain, in the limit,

$$
\lim _{\delta \downarrow}-m^{-1} \int_{\partial D}=\text { the value at } P \text { of the function } f \text {. }
$$

This we prove, in view of (3.6), by taking the local coordinates in such a way that $b_{i j}(0)=o_{i j}$-the geodesic coordinates at $P$. In this way, we have

$$
\int_{R} K_{m}(k, y)\left(\left(I-m^{-1} A\right) f\right)(y) d y=f(x)+\int_{R} L_{m}(x, y) f(y) d y,
$$

where

(3.10) $K_{m}(x, y)=\emptyset_{m}\left(r_{x, y}\right), r_{x, y}=$ the geodesic distance of $x$ and $y$,

and

$$
\begin{aligned}
L_{m}(x, y)= & \left(\left(I-m^{-1} A^{\prime}\right) K_{m}(x, y) \text { is infinitely differentiable for } x \neq y\right. \\
& \text { and is, in the vicinity of } x=y, \text { of the order } \\
& \begin{cases}r_{x, y}^{-1}, \quad(n=2), \\
r_{x, y}^{1-n}, \quad(n \geqslant 3) .\end{cases}
\end{aligned}
$$

$\S 4$. The integral representation of the resolvent $\boldsymbol{I}_{\boldsymbol{m}}$. We have, from (3.9),

$$
\left(I_{m} g\right)(x)+\int_{R} L_{m}(x, y)\left(I_{m} g\right)(y) d y=\int_{R} K_{m}(x, y) g(y) d y \text { for } g \in C(R) .
$$

This may be written as

$$
I_{m} g+L_{m} I_{m} g=K_{m} g
$$

Hence we have 


$$
\begin{gathered}
I_{m} g+L_{m}\left(K_{m} g-L_{m} I_{m} g\right)=K_{m} g, \text { that is, } \\
I_{m} g-L_{m}^{(2)} I_{m} g=\left(K_{m}-L_{m} K_{m}\right) g, \text { where } \\
\left(L_{m}^{(2)} g\right)(x)=\int_{R}\left\{\int_{R} L_{m}(x, z) L_{m}(z, y) d z\right\} g(y) d y, \\
\left(L_{m} K_{m} g\right)(x)=\int_{R}\left\{\int_{R} L_{m}(x, z) K_{m}(z, y) d z\right\} g(y) d y .
\end{gathered}
$$

Thus we obtain

$$
\begin{aligned}
& I_{m} g-L_{m}^{(2)}\left(L_{m}^{(2)} I_{m} g+K_{m}-L_{m} K_{m} g\right)=K_{m} g-L_{m} K_{m} g, \text { that is, } \\
& I_{m} g-L_{m}^{(4)} I_{m} g=\left(K_{m}-L_{m} K_{m}+L_{m}^{(2)} K_{m}-L_{m}^{(3)} K_{m}\right) g .
\end{aligned}
$$

Repeating the process, we obtain the integral equation of the form

$$
I_{m} g-L_{m}^{(k)} I_{m} g=\left(K_{m}-L_{m} K_{m}+\ldots\right) g \text {. }
$$

Because of (3.10) and (3.11), we may take $k$ so large that

(4.4) $M_{m}(x, y)=L_{m}^{(k)}(x, y)$ is continuous in $(x, y)$ and $N_{m}(x, y)=\left(K_{m}-L_{m} K_{m}+\ldots\right)(x, y)$ is continuous for $x \neq y$ and has the same order of singularity, for $x=y$, as $K_{m}(x, x)$.

We have thus proved that $\left(I_{m} g\right)(x)$ must satisfy the integral equation

$$
\left(I_{m} g\right)(x)-\int_{R} M_{m}(x, y)\left(I_{m} g\right)(y) d y=\int_{R} N_{m}(x, y) g(y) d y .
$$

By the continuity of the kernel $M_{m}(x, y)$, we may apply the classical theory of Fredholm to (4.5). Thus there exist a continuous kernel $Q_{m}(x, y)$ and $k^{\prime}$ functionals $c_{1}(g), c_{2}(g), \ldots, c_{k^{\prime}}(g)$ such that

$$
\begin{aligned}
\left(I_{m} g\right)(x) & =\int_{R^{i}} N_{m}(x, y) g(y) d \nu \\
& +\int_{R} Q_{m}(x, z) d z\left\{\int_{R} N_{m}(z, y) g(y) d y\right\}+\sum_{i=1}^{k^{\prime}} c_{i}(g) \varphi_{i}(x),
\end{aligned}
$$

where $\varphi_{1}(x), \varphi_{2}(x), \ldots, \varphi_{k^{\prime}}(x)$ form the linearly independent base of the solutions of the homogenous equations

$$
\int_{R} M_{m}(x, y) \varphi(y) d y=\varphi(x)
$$

Because of the lemmas 1-3, $\left(I_{m} g\right)(x)$ may, for fixed $x$, be considered as a bounded linear functional of $g \in C(R)$. Hence we have

$$
c_{i}(g)=\int_{R} \mu_{i}(d y) g(y),
$$

where $\mu_{i}$ are regular measures, countably additive for Borel sets $E$. These measures must, for sufficiently large $m$, be absolutely continuous with respect to the measure $d y$, and with bounded measurable densities: 


$$
\mu_{i}(E)=\int_{E} \nu_{i}(y) d y, \text { essential supremum }\left|\nu_{i}(y)\right|<\infty .
$$

This we see from the lemma 4, viz. from

$$
\lim _{s \rightarrow \infty} \int_{R}\left|h_{s}(x)\right| d x=0 \text { if } \lim _{s \rightarrow \infty} \int_{R}\left|\left(\left(I-m^{-1} A\right) h_{s}\right)(x)\right| d x=0 .
$$

Summing up, we have obtained the result: for sufficiently large $m$,

$$
\left(I_{m} g\right)(x)=\int_{R} p_{m}(x, y) g(y) d y, \quad g \in C(R),
$$

with a kernel $p_{m}(x, y)$ enjoying the conditions:

$$
\begin{aligned}
& p_{m}(x, y) \text { is measurable in }(x, y), \\
& p_{m}(x, y) \text { is continuous in } x \text { for fixed } y \neq x, \\
& p_{m}(x, y) \text { is, for } x=y, \text { of the same order as } K_{m}(x, y), \text { viz. } \\
& p_{m}(x, y\}=\left\{\begin{array}{l}
O\left(\log r_{x}, y\right), \quad n=2 \\
O\left(r_{x, y}^{2-n}\right), \quad n \geq 3 .
\end{array}\right.
\end{aligned}
$$

$\S 5$. An application to the stochastic processes. We will consider the special case of a symmetric operator $A$ :

$$
A=A^{\prime} \text {. }
$$

Since the singularity of the resolvent kernel $p_{m}(x, y)$ is given by (4.12), we see that its $k$-th iterated kernel $p_{m}^{(k)}(x, y)$ is, for sufficiently large $k$, a bounded measurable function of $(x, y)$. Thus, by Hilbert-Schmidt's expansion theorem, the Fourier series of the kernel $p_{m}^{(k)}(x, y)$ are absolutely and uniformly convergent on the product space $R \times R$. By virtue of this fact, we may prove ${ }^{5)}$ that the series

$$
\sum_{i=1}^{\infty} \frac{\varphi_{i}(x) \phi_{i}(y)}{\left(1-m^{-1} \lambda_{i}\right)^{k}}
$$

are, for sufficiently large $k$, absolutely and uniformly convergent on $R \times R$. Here $\left\{\psi_{i}(x)\right\}$ is a complete system of normal orthogonal eigenfunctions of the differential operator $A: \psi_{i}(x)$ belonging to the eigenvalue $\lambda_{i}$.

Proof. Let $\psi(x)$ be any eigenfunction of the operator $I_{m}$ :

$$
\left(I-m^{-1} \tilde{A}\right)^{-1} \psi=\mu \psi \text {. }
$$

We define, by the function $\psi(x)$, a distribution in the sense of Laurent Schwart $z{ }^{6)}$

$$
\emptyset(f)=\int_{R} \psi(x) f(x) d x, f \in D(A) .
$$

5) The same result is proved in other ways by K. Kodaira (unpublished) and by S. Minakshsundarum and A. Pleijel: - Some properties of the eigenfunctions of the Laplaceoperator on Riemannian manifolds, Canadian Journal of Math., Vol. 1, 242-256 (1950).

6) Schwartz: ibid. 
By virtue of (5.3), $\Phi$ satisfies the differential equation in the sense of the distribution:

$$
\left(I-m^{-1} A\right) \emptyset=\mu^{-1} \mathscr{D} .
$$

Since $\left(I-m^{-1} A\right)$ is elliptic, there exists ${ }^{7)}$ an infinitely differentiable function $\varphi(x)$ such that

$$
\left(\left(I-m^{-1} A\right) \varphi\right)(x)=\mu^{-1} \varphi(x), \varphi(x)=\psi(x)
$$

almost everywhere with respect to the measure $d x$.

Therefore we may assume $\psi(x)$ to be an eigenfunction of the differential operator $A$, belonging to the eigenvalue $m\left(1-\mu^{-1}\right)$ :

$$
(A \psi)(x)=m\left(1-\mu^{-1}\right) \psi(x) .
$$

It is easy to see that, conversely, any eigenfunction of (5.7), belonging to the eigenvalue $\lambda$, is also an eigenfunction of $\left(I-m^{-1} \tilde{A}\right)^{-1}$, viz. of the kernel $p_{m}(x$, $y)$, belonging to the eigenvalue $\left(1-m^{-1} \lambda\right)^{-1}$.

Therefore, by the absolute and uniform convergence of the Fourier series of the kernel $p_{m}^{(k)}(x, y)$, we see that the Fourier series (5.2) converge absolutely and uniformly on $R \times R$.

If we assume the negativity of the eigenvalues $\lambda$ of $A$, which is surely satisfied for the operator (5.11), we have

$$
\left(1-m^{-1} t \lambda_{i}\right)^{m} \leqq \exp \left(-\lambda_{i} t\right) \text { for } t>0 \text {. }
$$

Thus, by (5.2), the series

$$
\sum_{i=1}^{\infty} \exp \left(\lambda_{i} t\right) \psi_{i}(x) \phi_{i}(y)=P(t, x, y)
$$

are, for $i>0$, absolutely and uniformly convergent on $R \times R$.

\section{Let us assume further that}

$$
\int_{R} d x=1
$$

and

$$
(A f)(x)=\frac{1}{\sqrt{g(x)}} \frac{\partial}{\partial x^{i}}\left(\sqrt{g(x)} b^{i j}(x) \frac{\partial f}{\partial x^{j}}\right) .
$$

Then we may prove the probability condition

$$
P(t, x, y) \geqslant 0, \quad \int_{R} P(t, x, y) d y=1 .
$$

Proof. The last equality is proved by the orthonormality of $\left\{\psi_{i}(x)\right\}$ and the fact that we may take $\psi_{1}(x) \equiv 1$.

i) Schwartz: ibid. 
The proof of $P(t, x, y) \geqslant 0.8^{8)}$ We have, for

$$
f(t, x)=\int_{R} P(t, x, y) f(y) d y, \quad f(x)=\sum_{i=1}^{s} c_{i} \psi_{i}(x),
$$

the diffusion equation

$$
\frac{\partial f(t, x)}{\partial t}=A_{x} f(t, x)(t>0), \text { strong } \lim _{t \downarrow 0} f(t, x)=f(x) .
$$

Hence we have, for

$$
g_{\varepsilon}(t, x)=\exp (-\varepsilon t) f(t, x),
$$

the differential equation

$$
\frac{\partial g_{\varepsilon}(t, x)}{\partial t}=A_{x} g_{\varepsilon}(t, x)-\varepsilon g_{\varepsilon}(t, x), \quad g_{\varepsilon}(0, x)=f(0, x)=f(x) .
$$

Let $\varepsilon$ be $>0$ and let $g_{\varepsilon}(t, x)$ reach its minimum at the point $\left(t_{1}, x_{1}\right)$. Then we have

$$
\begin{aligned}
g_{\varepsilon}\left(t_{1}, x_{1}\right) & \rightleftharpoons \min _{\infty} f(x) \text { when } t_{1}=0 \\
& \geqslant 0 \text { when } t_{1}=\infty \text { or when } 0<t_{1}<\infty .
\end{aligned}
$$

The first two inequalities are evident. For, we have

$$
g_{\varepsilon}\left(0, x_{1}\right)=f\left(0, x_{1}\right) \supseteq \min _{x} f(x) \text { and } g_{\varepsilon}\left(\infty, x_{1}\right)=0
$$

Let $0<t_{1}<\infty$. Then, from

$$
\frac{\partial g_{\varepsilon}\left(t_{1}, x_{1}\right)}{\partial t}=0,\left(A_{x} g_{\varepsilon}\right)\left(t_{1}, x_{1}\right) \gtreqless 0 \text { and }(5.16) \text {, }
$$

we obtain $g_{\S}\left(t_{1}, x_{1}\right) \geqslant 0$. Thus we have (5.17) and hence, by letting $\varepsilon \downarrow 0$,

$$
f(t, x) \geqq \min \left(0, \min _{x} f(x)\right) \text {. }
$$

Therefore, by the denseness of $f(x)$ in $C(R)$, we must have $P(t, x, y) \geqslant 0$.

Q.E.D.

We have thus proved that, under the conditions (5.10) and (5.11), the series $P(t, x, y)$ give the explicite expression for the transition probability of the temporally homogeneous Markoff process, defined by the diffusion equation (5.14).

In concluding the paper, the author wishes to express his hearty thanks to Dr. Tosio Kato for his friendly criticism of the original manuscript.

\section{Mathematical Institute,}

\section{Nagoya University}

s) Cf. K. Yosida: Brownian motion on the surface of the 3-sphere, Ann. of Math. Statistics, Vol. 20, 292-296 (1949). 